\title{
Insuficiência cardíaca congestiva em crianças: do tratamento farmacológico ao transplante cardíaco
}

\author{
Heart failure in children: from the \\ pharmacologic treatment to \\ heart transplantation
}

\author{
Estela Azeka1, Luciana Marques de Vasconcelos², Tarcila \\ Marinho Cippiciani ${ }^{2}$, Adriana Santos de Oliveira ${ }^{2}$, \\ Denise Fabron Barbosa², Rafael Marcondes \\ Gonçalves Leite², Vânia Löschi Gapit ${ }^{2}$
}

\begin{abstract}
Azeka E, Vasconcelos LM, Cippiciani TM, Oliveira AS, Barbosa DF, Leite RMG, Gapit VL. Insuficiência cardíaca congestiva em crianças: do tratamento farmacológico ao transplante cardíaco. Rev Med (São Paulo). 2008 abr.-jun.;87(2):99-104.

RESUMO: A insuficiência cardíaca em crianças ocorre basicamente por: 1) defeitos cardíacos congênitos que levam à sobrecarga pressórica ou volumétrica na presença ou ausência de cianose; 2) cardiomiopatias congênitas ou adquiridas por erros inatos do metabolismo, distrofias musculares, infecções, drogas, toxinas e doença de Kawasaki; e 3) disfunção miocárdica após correção de defeitos cardíacos. Suas manifestações variam com a idade. Os sintomas mais comuns em lactentes são taquipnéia, taquicardia e dispnéia às mamadas; em crianças maiores fadiga e intolerância ao exercício; já em adolescentes são similares aos dos adultos. Seu tratamento pode ser a correção do defeito cardíaco congênito, com o uso ou não de drogas para otimizar o quadro clínico antes da cirurgia. Faz-se uso prolongado de medicação anti-congestiva em pacientes com defeitos cardíacos com tendência a fechamento espontâneo. As drogas utilizadas são digitálicos, diuréticos, inibidores da enzima conversora de angiotensina e os beta-bloqueadores. Estudos sobre eficácia das drogas mostram que a digoxina tem efeito benéfico modesto em crianças e os beta-bloqueadores melhoram a função ventricular. Há poucos estudos sobre a eficácia dos diuréticos e sobre os benefícios dos inibidores de angiotensina em crianças, principalmente se os inibidores possuem efeitos similares aos dos adultos e se apresentam efeitos no desenvolvimento da criança ao longo prazo. O transplante cardíaco tem sido indicado como tratamento principalmente para o estágio D de insuficiência cardíaca.
\end{abstract}

DESCRITORES: Insuficiência cardíaca/fisiopatologia. Insuficiência cardíaca/terapia. Insuficiência cardíaca/cirurgia. Diagnóstico clínico. Transplante de coração. Criança.

1. Médica Assistente da Unidade de Cardiologia Pediátrica e Cardiopatias Congênitas do Adulto do Instituto do Coração (InCor) do HCFMUSP. Conselheira da International Pediatric Transplantation Association (IPTA).

2. Acadêmicos da FMUSP em Medicina e integrantes da Liga de Insuficiência Cardíaca Congestiva e Transplante Cardíaco em Crianças, atividade de extensão da Faculdade de Medicina da Universidade de São Paulo.

Endereço para correspondência: Profa. Dra. Estela Azeka. Rua Araripina, 95. São Paulo, SP. CEP: 05603-030. e-mail: estela_azeka9@ hotmail.com 


\section{INTRODUÇÃO}

A insuficiência cardíaca na população pediátrica é uma entidade complexa apresentando peculiaridades devido às alterações fisiológicas em decorrência do próprio desenvolvimento cardíaco, múltiplas etiologias e associação com a correção operatória e/ou paliativa nos casos de defeitos cardíacos congênitos ${ }^{1}$.

Novos paradigmas têm sido descritos para explicar a natureza e progressão da Insuficiência Cardíaca Congestiva (ICC) baseados em estudos revelando interações complexas envolvendo fatores genéticos, celulares, neuro-hormonais e hemodinâmicos'.

A natureza congênita das alterações anatômicas, as alterações hemodinâmicas em decorrência dos defeitos cardíacos associados, sua relação com o tempo, isto é, início do aparecimento da ICC, momento da intervenção terapêutica são alguns dos aspectos relevantes à faixa etária pediátrica.

\section{Fisiopatologia e incidência}

A insuficiência cardíaca ${ }^{18}$ em crianças ocorre basicamente por: 1 - defeitos cardíacos congênitos que levam a sobrecarga pressórica ou volumétrica na presença ou ausência de cianose; 2 - cardiomiopatias congênitas ou adquiridas por erros inatos do metabolismo, distrofias musculares, infecções, drogas, toxinas e doença de Kawasaki, e 3 - disfunção miocárdica após correção de defeitos cardíacos. A causa mais freqüente de insuficiência cardíaca em lactentes e crianças são os defeitos cardíacos congênitos. A incidência de cardiopatia congênita na criança é de aproximadamente 8 por 1000 nascidos vivos, ou $0,8 \%$.

A incidência anual de insuficiência cardíaca em decorrência de defeitos congênitos é de aproximadamente 0,1 a $0,2 \%$ de nascidos vivos. Os defeitos mais freqüentes que causam a ICC incluem defeitos com desvio de sangue da esquerda para direita (como defeito do septo interventricular, defeito do septo atrioventricular, persistência do canal arterial, janela aorto-pulmonar, tronco arterioso comum); lesões obstrutivas esquerdas (como estenose aórtica crítica, coarctação de aorta severa, estenose mitral congênita) e insuficiência valvar atrioventricular ou semilunar congênita.

Em relação à incidência de cardiomiopatia ${ }^{22} \mathrm{em}$ lactentes e em crianças, há relato de prevalência de cardiomiopatia dilatada idiopática de 2,6 por 00.000 . O estudo de Baltimore-Washington revela incidência de cardiomiopatia no primeiro ano de vida de 4 casos por 100.000 nascidos vivos. O Registro de Cardio- miopatia Pediátrica realizado nos Estados Unidos relata incidência anual de 0,6 a 100.000 pessoas por cardiomiopatia em crianças de zero a 18 anos.

A prevalência total da insuficiência cardíaca em pacientes após correção do defeito congênito é desconhecida, sendo estimada entre 10 a $20 \%$ em pacientes após a cirurgia de Mustard ou Senning quando chegam na adolescência ${ }^{1,18}$.

Manifestações da insuficiência cardíaca congestiva em lactentes, crianças e adolescentes

Em lactentes ${ }^{25}$, os sintomas de insuficiência cardíaca mais comuns incluem taquipnéia, taquicardia, dispnéia as mamadas. Outros sinais são hepatomegalia, ritmo de galope no exame físico, e cardiomegalia, edema pulmonar na radiografia de tórax.

Crianças maiores podem exibir taquicardia e taquipnéia, porém a manifestação típica é fadiga e intolerância ao exercício; sendo a falta de apetite e dificuldade de crescimento e desenvolvimento freqüentes. Pode-se observar também distensão venosa e edema periférico. Adolescentes apresentam sintomas semelhantes aos adultos incluindo dispnéia, taquipnéia, fadiga, intolerância ao exercício, ortopnéia, dispnéia paroxística noturna e sintomas gastrintestinais.

A classificação modificada desenvolvida pela New York Heart Association ${ }^{15}$ é útil para quantificar a insuficiência cardíaca em crianças, bem como a escala de Ross ${ }^{25}$ para avaliar a insuficiência cardíaca em lactentes.

Tratamento da insuficiência cardíaca congestiva em lactentes, crianças e adolescentes

Em pacientes portadores de defeitos cardíacos congênitos estruturais, a correção do defeito cardíaco constitui-se na terapêutica de escolha. O digital, os diuréticos e inibidores da enzima conversora de angiotensina são utilizados temporariamente para otimizar a condição clínica dos pacientes antes da correção operatória, geralmente nas primeiras semanas ou meses de vida, isto é por um curto período de tempo. O uso prolongado da medicação anti-congestiva, limita-se a pacientes com defeitos cardíacos que apresentam tendência de fechamento espontâneo do defeito na evolução da história natural da doença como defeito do septo ventricular ou em pacientes portadores de defeitos congênitos cuja correção operatória deve aguardar o crescimento da criança como nos portadores de estenose mitral 
congênita, desde que o quadro clínico da criança permita e o risco de doença vascular pulmonar ou disfunção ventricular sejam mínimos ${ }^{1}$.

A terapêutica farmacológica específica para crianças portadoras de cardiomiopatia é escassa, como por exemplo, em pacientes portadores de erros inatos do metabolismo, embora a carnitina tem sido relatada como benéfica em alguns casos de pacientes portadores de deficiência da mesma; regimes dietéticos podem apresentar efeito benéfico em pacientes com deficiência enzimática específica. Doenças musculares como distrofia de Duchenne, doença mitocondrial e a maioria das doenças genéticas não possuem um tratamento específico etiológico. Manuseio dos sintomas com digital e diuréticos representam a base terapêutica na maioria destes pacientes.

Em pacientes com miocardite aguda, além do suporte terapêutico com drogas inotrópicas, ventilação mecânica, drogas antiarrítmicas e antitrombóticas têm sido descritos o uso de corticóides, imunoglobulinas e drogas imunossupressoras.

O tratamento de crianças, adolescentes e adultos jovens que apresentam sintomas de insuficiência cardíaca após correção de defeitos congênitos, inclui o uso de digital, diuréticos e inibidores da $\mathrm{ECA}^{1,15,18,22,24,25}$. Espironolactona ou beta-bloqueadores não tem sido utilizados consistentemente, embora há evidências de eficácia em adultos com insuficiência cardíaca devido a outras causas. Na maioria das vezes, a disfunção miocárdica perioperatória é transitória, com normalização da função posteriormente. No entanto, a ICC pode progredir em alguns pacientes imediatamente ou ao longo prazo sendo estes pacientes candidatos a transplante cardíaco.

\section{Evidência de eficácia de tratamento}

\section{Digoxina}

Nos Estados Unidos, a digoxina permanece como uma das principais drogas utilizadas na ICC, embora poucos estudos mostraram evidências da eficácia clínica tanto em adultos como em crianças. Estudos na literatura que avaliaram a eficácia terapêutica da digoxina em crianças mostram efeitos benéficos modestos ${ }^{7,19,24,29}$, porém estes estudos não foram randomizados ou duplo cego. Há evidências de aumento da atividade parassimpática cardíaca e de baroreceptores, com diminuição da atividade simpática central, exercendo efeito neuro-hormonal favorável. Devido aos riscos de intoxicação e limitações de evidência da eficácia de seu uso, estudos randomizados e controlados são necessários para determinar se a relação entre risco e benefício é positiva.

\section{Diuréticos}

Devido ao benefício clínico evidente dos tiazídicos e furosemide, poucos estudos tem sido realizados nos últimos anos em relação à eficácia destes agentes.

Estudo randomizado $7,16,19,24,29$ de avaliação da espironolactona em crianças demonstrou que a espironolactona é segura, com ação diurética eficaz, porém o impacto na mortalidade não foi avaliado.

\section{Inibidores da angiotensina}

Estudos realizados ${ }^{14,20,21,30}$ na literatura com inibidores da ECA forneceram pouca informação se crianças portadoras de insuficiência cardíaca por defeitos cardíacos congênitos poderiam se beneficiar como os adultos portadores de cardiomiopatia isquêmica. Pouco se sabe também sobre os efeitos ao longo prazo no desenvolvimento e crescimento somático em crianças na fase de crescimento que fizeram uso da medicação. Vários estudos ${ }^{14,20,21,30}$ têm mostrado efeito benéfico na estabilização e melhora clínica de lactentes portadores de ICC secundária a defeitos congênitos com desvio de sangue da esquerda para direita. Há estudos mostrando também redução dos marcadores neuro-hormonais ${ }^{14,20,21,30}$. Estudo retrospectivo mostrou redução da mortalidade em crianças portadoras de cardiomiopatia dilatada tratadas com inibidores da ECA comparada com o tratamento convencional (digoxina e diuréticos) ${ }^{20}$. Em pacientes após cirurgia de Fontan não se observou melhora hemodinâmica ou ao exercício após uso de dois meses de enalapril. Estudo recente prospectivo com inibidores da ECA imediatamente após anastomose cavopulmonar bidirecional, revelou diminuição significativa de derrame pleural na evolução destes pacientes.

\section{Beta-bloqueadores}

Estudos em adultos ${ }^{8}$ têm demonstrado efeito benéfico de beta-bloqueadores melhorando a fração de ejeção, reduzindo a mortalidade e necessidade de hospitalização com melhora da classe funcional NYHA e da tolerância ao exercício.

Em crianças, o uso de beta-bloqueadores ${ }^{11,27,28}$ tem mostrado melhora da função ventricular. Estudo $^{4}$ duplo-cego, randomizado que recrutou crianças 
portadoras de ICC grave candidatas a transplante cardíaco possibilitou retirada de pacientes da lista de transplante cardíaco. Estudos não randomizados $^{11,27,28}$ revelam melhora da função ventricular, da tolerância ao exercício e diminuição da necessidade de transplante cardíaco em pacientes com cardiomiopatia dilatada idiopática ou induzida por drogas.

Há relatos de uso do propranolol associado ao diurético e digoxina melhorando sintomas clínicos e reduzindo marcadores neuro-hormonais em lactentes com ICC por grande desvio de sangue esquerda para direita ${ }^{12}$.

O mecanismo de ação dos agentes betabloqueadores na ICC não está completamente estabelecido. Um dos mecanismos envolvidos consiste em prevenir e reverter disfunção ventricular e remodelação ventricular mediada por mecanismos adrenérgicos intrínsecos. Entretanto, o carvedilol apresenta propriedades adicionais (como o bloqueio alfa-adrenérgico, atividade antioxidante e antiendotelina) que apresentam capacidade de atenuar os efeitos adversos do sistema nervoso simpático na circulação. Estes mecanismos de ação adicionais podem ser particularmente importantes na ICC grave e determinar diferenças entre os efeitos do carvedilol e outros beta-bloqueadores como o buncidolol.

Apesar de diferenças nos mecanismos fisiopatológicos da ICC em crianças, particularmente no neonato quando comparado com o adulto, envolvendo mecanismos celulares de regulação do cálcio no acoplamento da excitação e contração relacionados aos receptores beta-adrenérgicos, o carvedilol melhorou sintomas e função ventricular nestes pacientes.

As estratégias no tratamento da ICC crônica em crianças candidatas ao transplante cardíaco têm tradicionalmente seguido as recomendações do tratamento de ICC dos pacientes adultos ${ }^{6}$. No entanto, o fato da etiologia, fisiopatologia da ICC em crianças freqüentemente apresentar características próprias, justifica-se o desenvolvimento de estudos randomizados, prospectivos para melhor avaliar o tratamento medicamentoso da ICC nesta faixa etária. Estes dados fornecerão informações que serão úteis no manuseio apropriado, otimizando o tratamento medicamentoso da ICC em crianças. O transplante cardíaco que tem sido a opção terapêutica em crianças portadoras de ICC grave ${ }^{3,13}$, porém apresenta limitações pela escassez de potenciais doadores, portanto estratégias terapêuticas que possam diminuir a fila de espera ou mesmo postergar a necessidade de transplante cardíaco permanecem o objetivo principal neste grupo de paciente ${ }^{10}$.

\section{Transplante cardíaco}

Em 1967, era realizado, na África do Sul ${ }^{6}$, o primeiro transplante cardíaco entre humanos e, no mesmo ano, era relatado o primeiro transplante neonatal, quando Kantrowitz ${ }^{17}$, tentou, sem sucesso, o transplante em recém-nato portador de anomalia de Ebstein.

A aplicação clínica do transplante, porém, devese ao pioneirismo de Bailey que, em $1984^{5}$ realizou o primeiro xenotransplante em criança portadora de síndrome da hipoplasia do ventrículo esquerdo e, um ano após, realizou o primeiro transplante em recémnato com a mesma cardiopatia.

Desde então, o transplante tem sido adotado como opção terapêutica. Todavia, foi somente anos mais tarde, com a introdução da ciclosporina ${ }^{23}$ como droga imunossupressora de base que houve um alento motivando o reinício da atividade dos grupos que realizavam transplante.

O transplante cardíaco tem sido indicado como tratamento ${ }^{2}$ para o estágio $D$ de insuficiência cardíaca (segundo classificação NYHA), no qual o paciente tem sintomas persistentes mesmo em repouso, podendo necessitar de infusão continua de drogas vasoativas e/ou ventilação mecânica e/ou suporte circulatório mecânico. Dados mostram que pacientes em estágio $\mathrm{D}$ têm alto risco de morte iminente e que pode ser eficientemente evitado com transplante cardíaco.

Os pacientes em estágio $C$ de insuficiência cardíaca apresentam sintomas, mas não são dependentes de inotrópicos, ventilação e/ou circulação mecânica. Estes pacientes podem apresentar piora do quadro enquanto estão na fila, evoluindo para o estágio D.

A presença de arritmias que ameaçam a vida do paciente pediátrico e que não pode ser tratada por medicação ou implantação de desfibriladores representa outra indicação para transplante, não importando o estágio da insuficiência.

Na última década do século passado, observouse aumento progressivo do número de transplantes cardíaco infantis, isto paralelamente ao desenvolvimento de novas abordagens terapêuticas, como o uso de beta-bloqueadores para insuficiência cardíaca e com o desenvolvimento de novas técnicas e indicações cirúrgicas, como procedimentos paliativos para a hipoplasia do ventrículo esquerdo. Como conseqüência destes avanços terapêuticos, outros dois aspectos tornaram-se importantes: a indicação de transplante para pacientes adultos com cardiopatias congênitas e a indicação de retransplante para paciente que recebera o primeiro enxerto na infância.

Apesar do sucesso do transplante cardíaco 
infantil, este apresenta co-morbidades ${ }^{2,26}$ e também tem surgido o questionamento quanto à necessidade de retransplante nestes pacientes. Alguns estudos demonstram que o transplante está relacionado a uma mortalidade anual que chega a $2 \%$ a $3 \%$, porém com $70 \%$ de chance de ocorrência de eventos como rejeição, doença vascular do enxerto ou ambos ao longo prazo ${ }^{9}$.

O fato é que a sobrevida, ao longo prazo do enxerto, é particularmente importante em se tratando de transplante cardíaco, pois os sinais da falência do enxerto poderão se manifestar quando o paciente estiver na adolescência ou na vida adulta, ou seja, em períodos de maior atividade produtiva destes indivíduos. Segundo dados do Registro da ISHLT, há incidência da doença vascular do enxerto que chega a cerca de $20 \%$ em 10 anos, sendo um dos principais fatores limitantes da sobrevida. Portanto, estudos são necessários na prevenção e terapêutica desta complicação ao longo prazo ${ }^{9}$.

Azeka E, Vasconcelos LM, Cippiciani TM, Oliveira AS, Barbosa DF, Leite RMG, Gapit VL. Heart failure in children: from the pharmacologic treatment to heart transplantation. Rev Med (São Paulo). 2008 abr.-jun.;87(2):99-104.

ABSTRACT: Heart failure in children occurs basically because of (1) congenital heart defects that lead to pressure or volume overload in the presence or absence of cyanosis; (2) congenital or acquired cardiomyopathies by innate errors in metabolism, muscular dystrophies, infections, drugs, toxins and Kawasaki disease; and (3) myocardial dysfunction after correction of cardiac defects. Its manifestation varies with age. The most common symptoms in infant are tachycardia, tachypnea and dyspnea while suckling; in older children, fatigue and intolerance to exercise; in the adolescents the symptoms are similar to those in the adults. Its treatment can be the correction of the cardiac defect with or without use of drugs to optimize the clinical condition before the surgery. Extended use of anticongestive medication is used in patients with cardiac defects that tend to amend spontaneously. The drugs utilized are digoxin, diuretics, angiotensin inhibitors and beta-blockers. Studies about drugs' efficacy suggest that digoxin has a modest beneficial effect in children and beta-blockers improve ventricular function. There are few studies about the efficacy of diuretics and about the benefits of angiotensin inhibitors in children, mainly if the $\mathrm{ACl}$ has similar effects of adults and if they may affect the child's growth in long-term period. Heart transplantation has been indicated principally as a treatment for stage $D$ heart failure.

KEY WORDS: Heart failure/surgery. Heart failure/physiopathology. Heart failure/therapy. Diagnosis, clinical. Heart transplantation. Child.

\section{REFERÊNCIAS}

1. Auslender M. Pathophysiology of pediatric heart failure. Prog Pediatr Cardiol. 2000;11:175-84.

2. Azeka E, Loures DR, Jatene M, Favarato ME. I Diretriz Brasileira de Transplante Cardíaco: indicação, evolução tardia, aspectos peculiares, doador e avaliação e suporte psicológico em transplante cardíaco em crianças. Arq Bras Cardiol. 1999;73(Suppl 5): 6-11.

3. Azeka E, Marcial MB, Jatene M, Auler JO Jr, Ramires JA. Eight-year experience of pediatric heart transplantation: clinical outcome using non-invasive methods for the evaluation of acute rejection. Pediatr Transplant. 2002;6: 208-13.

4. Azeka E, Ramires JA, Valler C, Bocchi E. Delisting of infants and children from the heart transplantation waiting list after carvedilol treatment. J Am Coll Cardiol. 2002:40:2034-8.

5. Bailey LL, Nehlsen-Cannarella SL, Doroshow RW, Jacobson JG, Martin RD, Allard MR, et al. Cardiac allotransplantation in newborns as therapy for hypoplastic left heart syndrome. $\mathrm{N}$ Engl $\mathrm{J}$ Med. 1986;315:949-51.

6. Barnard CN. A human cardiac transplant: an interim report of a successful operation performed at Groote Schurr Hospital, Cape Town. S Afr Med J. 1967; 41:1271-4.

7. Berman W Jr, Yabek SM, Dillon T, Niland C, Corlew $S$, Christensen D. Effects of digoxin in infants with congestive circulatory state due to a ventricular septal defect. N Engl J Med. 1983; 308:363-6.

8. Bocchi EA, Bacal F, Bellotti G, Carrara D, Ramires JA. Effects of carvedilol (beta 1, beta 2, alpha 1 blocker) on refractory congestive heart failure. Arq Bras Cardiol. 1998;71:169-73.

9. Boucek MM, Aurora P, Edwards LB, Taylor DO, Trulock EP, Christie J, et al. Registry of International Society for Heart and Lung Transplantation: tenth official pediatric heart transplantation report -2007 . J Heart Lung Transplant, 2007;796-805. 
10. Boucek MM. Breaching the barrier of $A B O$ incompatibility in heart transplantation for infants. N Engl J Med. 2001; 44:843-4.

11. Bruns LA, Chrisant MK, Lamour JM, Shaddy RE, Pahl $\mathrm{E}$, Blume ED, et al. Carvedilol as therapy in pediatric heart failure: An initial multicenter experience. J Pediatr. 2001;138:505-11.

12. Buchhorn R, Bartmus D, Siekmeyer W, HulpkeWette M, Schulz R, Bürsch J. Beta-blocker therapy of congestive heart failure in infants with left to right shunts. Am J Cardiol. 1998;81:1366-8.

13. Canter CE, Naftel DC. Recipient characteristics. In: Fine R, Webber S, Harmon W, Olthoff, K, Kelly D, editors. Pediatric solid organ transplantation. 2nd ed. Malden, Mass: Blackwell; 2007. Chap. 31.

14. Cohn JN, Johnson G, Ziesche S, Cobb F, Francis $G$, Tristani $F$, et al. A comparison of enalapril with hydralazine-isosorbide dinitrate in the treatment of chronic congestive heart failure. $\mathrm{N}$ Engl $\mathrm{J}$ Med. 1991;325:303-10.

15. Connolly D, Rutkowski M, Auslender M, Artman M. The New York University Pediatric Heart Failure Index: a new method of quantifying chronic heart failure severity in children. J Pediatr. 2001;138:644-8.

16. Hobbins SM, Fowler RS, Rowe RD, Korey AG. Spironolactone therapy in infants with congestive heart failure secondary to congenital heart disease. Arch Dis Child. 1981;56:934-8.

17. Kantrowitz A, Haller JD, Joos H, Cerruti MM, Carstensen HE. Transplantation of the heart in an infant and an adult. Am J Cardiol. 1968;22:782-90.

18. Kay JD, Colan SD, Graham TP, Durbam NC. Congestive heart failure in pediatric patients. Am Heart J. 2001;142:923-8.

19. Kimball TR,Daniels SR,Meyer RA,Hannon DW,Tian J,Shukla R, et. al. Effect of digoxin on contractility and symptoms in infants with large ventricular septal defect. Am J Cardiol. 1991; 68:1377-82.

20. Latson LA. Captopril in children with cardiomyopathies. Circulation. 1991;83:707-8.

21. Lewis $A B$, Chabot $M$. The effect of treatment with angiotensin-converting enzyme inhibitors on survival of pediatric patients with dilated cardiomyopathy. Pediatr Cardiol. 1993;14:9-12.

22. Lipshultz SE, Sleeper LA, Towbin JA, Lowe AM, Orav EJ, Cox GF, et al. The incidence of pediatric cardiomyopathy in two regions of the United States. N Engl J Med. 2003;348:17.

23. Oyer PE, Stinson EB, Jamieson SW, et al. Cyclosporine in cardiac transplantation. A $21 / 2$ year follow up. Transplant Proc. 1983;15:2546-52.

24. Rosenthal D, Chrisant MR, Edens E, Mahony L, Canter C, Colan S, et al. International Society for Heart and Lung Transplantation: practice guidelines for management of heart failure in children. $J$ Heart Lung Transplant. 2004;23:1313-33.

25. Ross RD, Bollinger RO, Pinsky WW. Grading the severity of congestive heart failure in infants. Pediatr Cardiol. 1992;13:72-5.

26. Schowengerdt KO, Azeka E. Infection following pediatric transplantation. In: Canter CE, Kirklin JK. ISHLT monograph series: pediatric heart transplantation. Elsevier; 2007. v.2. Chap.10:157-71.

27. Shaddy RE. Beta-blocker therapy in young children with congestive heart failure under consideration for heart transplantation. Am Heart J. 1998;136:19-21.

28. Shaddy RE, Tani LY, Gidding SS, Pahl E, Orsmond GS, Gilbert EM, et al. Beta-blocker treatment of dilated cardiomyopathy with congestive heart failure in children: a multi-institutional experience. J Heart Lung Transplant. 1999;18:269-74.

29. Seguchi M, Nakazawa M, Momma K. Further evidence suggesting a limited role of digitalis in infants with circulatory congestion secondary to large ventricular septal defect. Am J Cardiol. 1999;83:1408-11.

30. Study of Left Ventricular Disease (SOLVD) Investigators. Effect of enalapril on survival in patients with reduced left ventricular ejection fractions and congestive heart failure. N Engl J Med. 1991;325:293-302.

31. West LJ, Pollock-Barziv SM, Dipchand AI, Lee KJ, Cardella CJ, Benson LN, et al. ABO-incompatible heart transplantation in infants. $\mathrm{N}$ Engl J Med. 2001;344:793-800. 